\title{
Complex Fragments from Excited Actinide Nuclei: A New Test of the Finite Range Model
}

D.G. Sarantities, O.R. Bowman, G.J. Wozniak, R.J. Charity, Z.H. Liu, R.J. McDonald, M.A. McMahan and L.G. Moretto

Nuclear Science Division

Lawrence Berkeley Laboratory

University of California

Berkeley, California 94720

May 1988

\section{DISCLAIMER}

This report was prepared as an account of work sponsored by an agency of the United States Government. Neither the United States Government nor any agency thereof, nor any of their employees, makes any warranty, express or implied, or assumes any legal liability or responsibility for the accuracy, completeness, or usefulness of any information, apparatus, product, or process disclosed, or represents that its use would not infringe privately owned rights. Reference herein to any specific commercial product, process, or service by trade name, trademark, manufacturer, or otherwise does not necessarily constitute or imply its endorsement, recommendation, or favoring by the United States Government or any agency thereof. The views and opinions of authors expressed herein do not necessarily state or reflect those of the United States Government or any agency thereof.

This work was supported by the Director, Office of Energy Research, Division of Nuclear Physics of the Office of High Energy and Nuclear Physics of the U.S. Department of Energy under Contract DE-AC03-76SF00098. 


\title{
Complex Fragments from Excited Actinide Nuclei:
}

\section{A New Test of the Finite Range Mode!}

\author{
by \\ D. G. Sarantities \\ Department of Chemistry, Washington University, St. Louis, \\ Missouri, 63130 \\ and
}

D. R. Bowman, G. J. Wozniak, R. J. Charity, Z. H. Liu ${ }^{(a)}$, R. J. McDonald,

M. A. McMahan, and L. G. Moretto

Nuclear Science Division, Lawrence Berkeley Laboratory, University of California, Berkeley,

California, 94720

Complex fragments ranging in charge from $7 \leq \mathrm{Z} \leq 45$ have been detected in binary coincidence following the reaction of $8.4 \mathrm{MeV} / \mathrm{u}{ }^{232} \mathrm{Th}+{ }^{12} \mathrm{C}$, and are shown to arise from the binary decay of a ${ }^{244} \mathrm{Cm}$ compound nucleus. This work confirms earlier radiochemical observations of very light fragments in the fission fragment mass distribution, establishes their binary character, and interprets their yield in terms of funite range potential energy barriers. 
Complex fragment radioactivity has been found recently in actinide and preactinide nuclei. ${ }^{\text {: }}$ The interpretation of the associated half-lives hinges on the simultaneous understanding of both the potential energy and the inertia tensor in a relevant set of collective coordinates. The modulation of both quantities by shell effects is to a large extent unknown but is expected to be large enough to influence profoundly the decay rate. Therefore it is desirable on one hand to isolate the role of the potential energy from that of the inertia tensor, and on the other to minimize the shell effects in order to reveal the underlying smooth liquid drop-like potential. It is possible to achieve both goals by studying the same channels in compound nucleus decay, since the decay rate depends mainly upon the height of the barrier and, at sufficiently high energies the shell effects are substantially reduced.

Early radiochemical measurements of the inclusive cross-sections following the reaction of $30.6 \mathrm{MeV}{ }^{3} \mathrm{He}$ and ${ }^{238} \mathrm{U}$ observed, in addition to the expected fission peak near symmetry, detectable yields of products with masses between those of alpha particles and classical "fission fragments" (see Fig. 1 in Ref. 2). Those results, along with other similar work ${ }^{3,4}$, were not understood at the time, and the production of fragments with $10<Z<17$ was ascribed to a ternary fission mechanism.

Recent experimental studies have proven unquestionably that binary compound nucleus decay is a source of complex fragments (CFs), both at low incident energies ${ }^{5-7}$, and continuing up to beam energies of $50 \mathrm{MeV} / \mathrm{u}^{8,9}$ In these studies, compound systems with masses between $A=100$ and 150 have been investigated. However, theoretical arguments predict that the emission of CFs should also be observed from heavier compound nuclei. Thus, it is reasonable to expect that the fragments observed in ref. 2 may arise from binary compound nucleus decay.

The compound nucleus emission of complex fragments was predicted by generalizing the compound nucleus decay theory so that it incorporates the mass asymmetry coordinate. ${ }^{10}$ In this model all of the possible decay channels associated with the various exit channel asymmetries compete statistically so that fission and light particle evaporation are seen as the extremes of a continuous process rather than two independent decay modes. The emission probability $\left(\Gamma_{z} / \hbar\right)$ at a given mass asymmetry $\left(\mathrm{Z} / \mathrm{Z}_{\mathrm{CN}}\right)$ is determined by the conditional barrier $\left(\mathrm{B}_{2}\right)$ and the saddle point temperature $\left(\mathrm{T}_{\mathrm{z}}\right)$.

$$
\Gamma_{z} \equiv \frac{T_{z}}{2 \pi} \frac{\rho\left(E-B_{z}\right)}{\rho(E)}-\frac{T_{z}}{2 \pi} e^{-B_{z} / T_{z}}
$$


As stated above, this emission probability is independent of the inertia.

Figure 1 illustrates how both the conditional barriers and the relative yields depend on the mass asymmetry of the exit channel for the ${ }^{244} \mathrm{Cm}$ compound nucleus. There are three local minima in the potential energy curve (corresponding to the maxima in the yield) at asymmetries of $0.0,0.5$, and 1.0 and two maxima (corresponding to minima in the yield), called the Businaro-Gallone mountains whose location depends upon the fissility parameter of the system. From this figure one can see that fission (symmetric decay), light particle evaporation (very asymmetric decay), and the much less probable complex fragment emission (decay at intermediate asymmetries) are all manifestations of the same process.

In order to verify that the light complex fragments observed in ref. 2 are originating from binary decay and not from ternary fission, and to investigate the role of the potential energy on the decay rate, we have recently studied a very similar system at a somewhat higher excitation energy. The system studied in this work is $8.4 \mathrm{MeV} / \mathrm{u}^{232} \mathrm{Th}+{ }^{12} \mathrm{C}$, leading to the compound nucleus ${ }^{244} \mathrm{Cm}$ with $70 \mathrm{MeV}$ of excitation energy.

We have chosen to study this reaction in reverse kinematics because of the ease with which heavy fragments can be detected. The large velocity of the source produced with heavy beam nuclei incident upon light target nuclei has the advantage of producing reaction products with large laboratory velocities, which allow for the easy detection and identification of the heavy fragments. As an added advantage, the kinematic focusing of the emitted fragments greatly increases both the inclusive and coincidence detection efficiencies.

An $8.4 \mathrm{MeV} / \mathrm{u}{ }^{232} \mathrm{Th}$ beam from the Lawrence Berkeley Laboratory SuperHILAC bombarded a $1.01 \mathrm{mg} / \mathrm{cm}^{2}{ }^{12} \mathrm{C}$ target. Light fragments were detected with an $\mathrm{X}-\mathrm{Y}$ position sensitive $\left( \pm 0.5^{\circ}\right)$ gas - silicon telescope situated at $20^{\circ}-29^{\circ}$ in the laboratory. With this detector, individual charge identification up to a $Z$-value of $\mathbf{4 5}$ was achieved. The coincident heavy fragments were detected with a $20 \times 30 \mathrm{~cm}^{2} \mathrm{X}-\mathrm{Y}$ position sensitive $\left( \pm 0.1^{\circ}\right)$ multiwire avalanche detector (PPAC) spanning a laboratory range of $3^{\circ}-17^{\circ}$ in plane.

The method of analysis was as follows: after the initial two-body decay, the secondary (post-evaporative) values $Z_{3}, E_{3}, \theta_{3}{ }^{\prime}$, and $\phi_{3}{ }^{\prime}$ were measured for the light fragment in the $\Delta E-E$ telescope. Corrections to the measured energy were made to take into account the energy losses in the target, absorber foils, and ion chamber window. The fragrnent mass at emission, $A_{3}$ was calculated assuming charge equilibration (minimization of the potential energy at scission). From $A_{3}, E_{3}, \theta_{3}$, and $\phi_{3}$ the quantities $A_{4}, E_{4}, \theta_{4}$, and $\phi_{4}$ were calculated via two-body kinematics (to first order, the primary emission angles $\theta_{3}$ and $\phi_{3}$ will not change with sequential light particle 
emission). An iterative evaporation calculation was then performed assuming a cost of $12 \mathrm{Me} !$ of excitation energy per evaporated neutron until consistent values of $E_{3}$ and $A_{3}$ wete obrained.

Finally from the primary values $Z_{3}, E_{3}, \theta_{3}$, and $\phi_{3}$, the corresponding values $Z_{4}, E_{4}, \theta_{4}$, and $\phi_{4}$ could be calculated for the heavy fragment. A comparison of the calculated values of $\theta_{4}$, and $\phi_{4}$ with the angles observed in the avalanche counter allows one to verify if the physical events do in fact arise from the binary decay of the ${ }^{244} \mathrm{Cm}$ compound nucleus.

In Figure 2 a correlation plot of $\Delta \phi\left(\phi_{4 \text { observed }}-\phi_{4 \text { calculated }}\right)$ vs. $\Delta 6\left(\theta_{4 \text { observed }}-\theta_{4 \text { calculated }}\right)$ is shown for four representative $Z$-values of 26, 22, 20 and 13 detected in the ionization chamber, corresponding to progressively more asymmetric decay. The events resulting from binary decay should be clustered about $\Delta \phi=0^{\circ}$ and $\Delta \theta=0^{\circ}$. The region enclosed by the elliptical line is centered about this value and delineates the expected spread in $\phi$ and $\theta$ due to beam spot size, position resolution, and sequential evaporation (neutron and possibly light charged particle) from the primary fragments $Z_{3}$ and $Z_{4}$. It is clear in these cases from the tight distribution around $\Delta \phi=0^{\circ}$ and $\Delta \theta=0^{\circ}$ that the bulk of these data can be explained as originating from a two-body decay of the initial system.

Figure 3 illustrates the charge distribution of the events characterized as binary in this manner. Note the similarity in shape to the radiochemical mass distribution (Fig 1 in Ref. 2). The present results indicate that the binary component extends to very large asymmetries and verifies the inherent unity of fission, complex fragment emission, and evaporation.

The observed increase in yield for Z-values $<16$ in Fig. 3 can be explained qualitatively in terms of the standard liquid drop model. In the model (see Fig. 1), the potential energy as a function of mass asymmetry has local minima at asymmetries of $0.0,0.5$ (symmetry), and 1.0, and local maxima at intermediate asymmetries. The exit channel asymmetry with the largest potential energy banier should have the minimum yield. Therefore the liquid drop model predicts that the yields will increase on both sides of the maxima.

Due to the special stability of the doubly magic ${ }^{208} \mathrm{Fb}$ nucleus, there are also shell effects that may influence the yields. These effects are responsible for the spontaneous ${ }^{14} \mathrm{C}$, and $\mathrm{Ne}$ isotope radioactivities recently observed from actinide and preactinide nuclei. ${ }^{1}$ However in the present case shell effects may not be so important because of the sizable excitation energy. ${ }^{11}$

In figure 1 the $\mathrm{I}=0$ conditional barriers were calculated from the liquid drop model ${ }^{12}$, and the relative yields were determined assurning a Fermi gas level density with level density parameters $a_{\mathrm{f}}=\mathrm{a}_{\mathrm{z}}=\mathrm{A} / 10 \mathrm{MeV}^{-1}$. In the two regions between the asymmetries of 0.07 and $n 425$, and 0.575 
and 0.93 no constrained saddle points are found in the liquid drop model due to the near sphericity of the system. The yields in this region have been estimated by simply drawing a smooth curve between the regions where the conditional barriers do exist.

From inspection of the calculated yields in Fig. 1, one observes a factor of approximately $10^{10}$ between the maximum and minima of the distribution, where constrained saddle points exist. The experimentally observed distribution (Fig. 3) shows only a factor of $10^{5}$ between the maximum and minimum yields. Even allowing for the roughness of the calculation and the uncertainties due to angular momentum, this is still a huge discrepancy between theory and experiment.

This disagreement can be removed by the inclusion of the finite range effect ${ }^{13}$ into the calculation of the potential energy. The finite range effects arise from the surface-surface interaction which is particularly important for the very indented shapes near the Businaro-Gallone mountains, but almost unimportant at symmetry as shown in fig. 1 . Therefore the inclusion of these effects has very little effect on the conditional barriers near symmetry of a nucleus with a large fissility parameter. However, it can have very large effects on the asymmetric barriers. For ${ }^{244} \mathrm{Cm}$ the inclusion of finite range effects dramatically lowers the barriers at mass asymmetries near the maximum potential energy, but hardly changes the symmetric barrier ${ }^{14}$ (see Fig. 1). The ratio of the maximum/minimum relative yields recalculated by incorporating finite range effects is about $10^{5}$, in excellent agreement with the experimentally observed ratio (see Fig. 3). The influence of finite range effects on the mass asymmetric barriers in lighter systems has been previously observed. ${ }^{15}$

In conclusion, it appears that for the entire mass distribution observed in the reaction of 8.4 $\mathrm{MeV} / \mathrm{u}^{232} \mathrm{Th}+{ }^{12} \mathrm{C}$, the primary production mechanism is the binary decay of the ${ }^{244} \mathrm{Cm}$ compound nucleus. The ratio of the yields of symmetric/asymmetric products cannot be explained within the standard liquid drop model, but can be explained to better than one order of magnitude with the inclusion of finite range effects. ${ }^{14}$ In essence the entire range of products (evaporation residues, fission fragments, complex fragments, and evaporated light particles) originates from the same mechanism, the statistical, binary decay of an excited compound nucleus. These data demonstrate the inherent unity of fission, complex fragment emission, and light particle evaporation which was predicted theortetically in $1975^{10}$.

This work was supported by the Director, Office of Energy Research, Division of Nuclear Physics of the Office of High Energy and Nuclear Physics of the U. S. Department of Energy under Contracts DE-AC03-76SF00098 and DE-AS02-76ER04052.

\section{References}

(a) Present address: Institute of Atomic Energy, Beijing, China. 
1. H. J. Rose and G. A. Jones, Nature, 307, 245 (1984), S. Gales, et al., Phys. Rev. Lett. 53, 759 (1984), P.B. Price et al., Phys. Rev. Lett. 54, 297 (1985), S. Barwick et al., Phys. Rev. C34, 362 (1986).

2. K. W. MacMurdo and J. W. Cobble, Phys. Rev. 182, 1303 (1969).

3. R. H. Iyer and J. W. Cobble, Fhys. Rev. Lett. 17, 541 (1966).

4. R. H. Iyer and J. W. Cobble, Phys. Rev. 172, 1186 (1968).

5. L. G. Sobotka et al., Phys. Rev. Lett. 51, 2187 (1983).

6. L. G. Sobotka et al., Phys. Rev. Lett. 53, 2004 (1984).

7. M. A. McMahan et al., Phys. Rev. Lett. 55, 1995 (1985).

8. R. J. Charity et al., Phys Rev. Lett. 56, 1354 (1986).

9. D. R. Bowman et al., Phys. Lett. 189B, 282 (1987).

10. L. G. Moretto, Nucl. Phys. A247, 211 (1975).

11. L. G. Moretto, Nucl. Phys. A182, 641 (1972).

12. K. T. R. Davies and A. J. Sierk, Phys. Rev. C31, 915 (1985).

13. H. J. Krappe et al., Phys. Rev. C20, 992 (1979).

14. A. J. Sierk, private communication.

15. Amold J. Sierk, Phys. Rev. Lett. 55, 582 (1985). 


\section{Figure Captions}

Figure 1. The liquid-drop potential energy surface (heavy line) and the corresponding yields

(dotted line) as a function of the fragment mass asymmetry $\left(Z_{\text {trag }} / Z_{C N}\right)$ for the ${ }^{244} \mathrm{Cm}$ compound nucleus. The dashed section between the asymmetries of $.07(.93)$ and $.425(.575)$ indicates the region where no constrained saddle points exist due to the near sphericity of the system ${ }^{12}$ (in this region the barriers and the corresponding yields have been interpolated smoothly). The vertical arrows indicate the lowering of the calculated barriers at symmetry and at the Businaro-Gallone mountains due to finite range effects.

Figure 2. A comclation plot of the deviations $\Delta \phi\left(\phi_{4 \text { observed }}-\phi_{4 \text { calculated }}\right)$ vs. $\Delta \theta\left(\theta_{4 \text { observed }}{ }^{-}\right.$ $\theta_{4 \text { calculated }}$ ) of the observed minus the calculated in and out-of-plane recoil angles for four representative Z-values of $26,22,20$, and 13 (see text). Events due to binary decay show clustering around the point $\Delta \theta=0, \Delta \phi=0$. The region enclosed by the solid line delineates the expected spread in $\Delta \theta$ and $\Delta \phi$ due to beam spot size, position resolution, and sequential light particle evaporation.

Figure 3. Relative yields of the binary events as a function of the Z-value of the light fragment defected in the ionization chamber. These events were characterized as binary by the detection in coincidence of a recoil fragment at the angles $\theta$ and $\phi$ predicted by two-body kinematics. The arrow indicates the theoretical ratio of the yield at symmetry to that at the Businaro-Gallone mountains determined from the finite range model. 


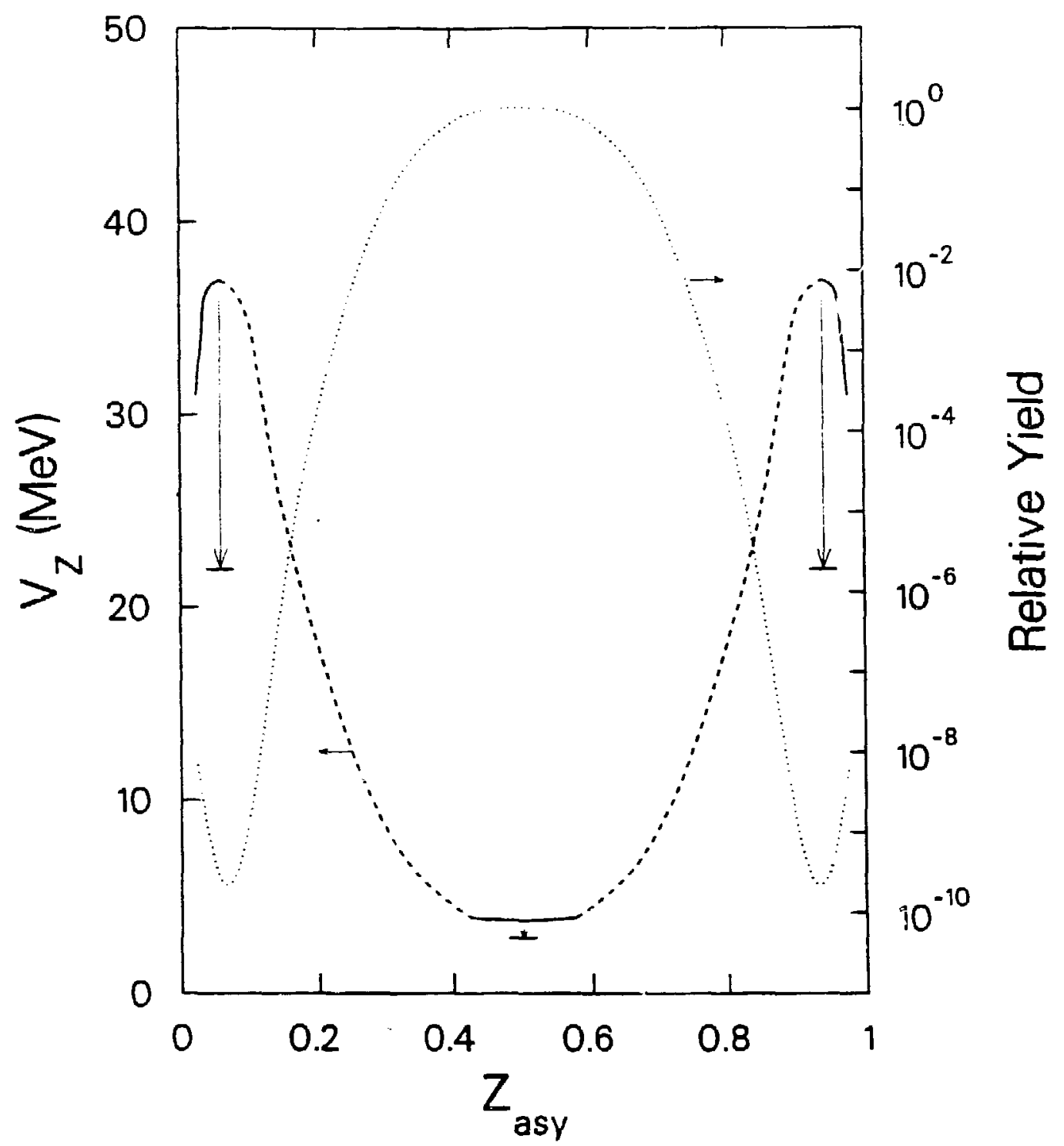

XBL $884-1450$

Fi.g. 1 


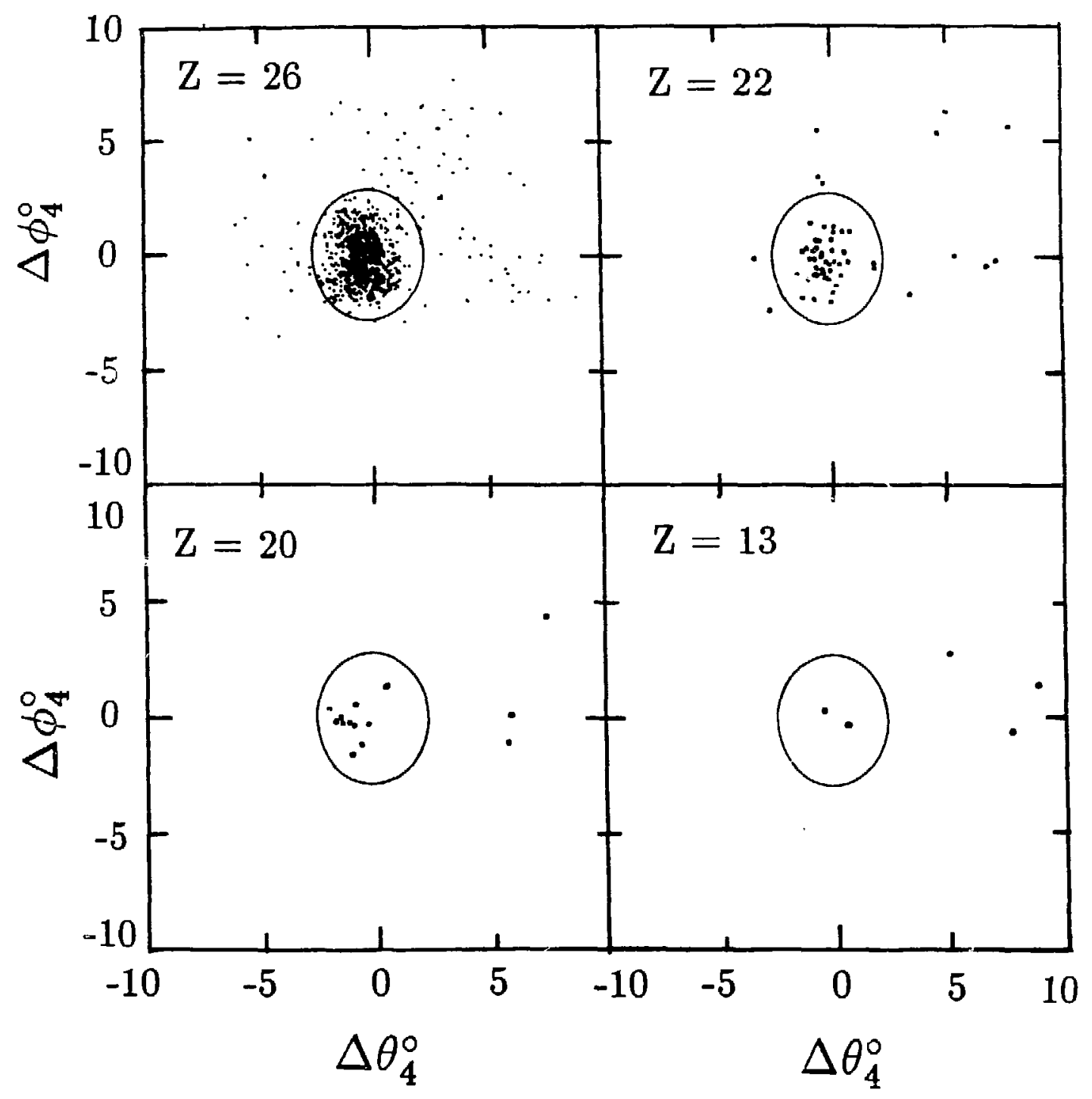

Fig. 2 


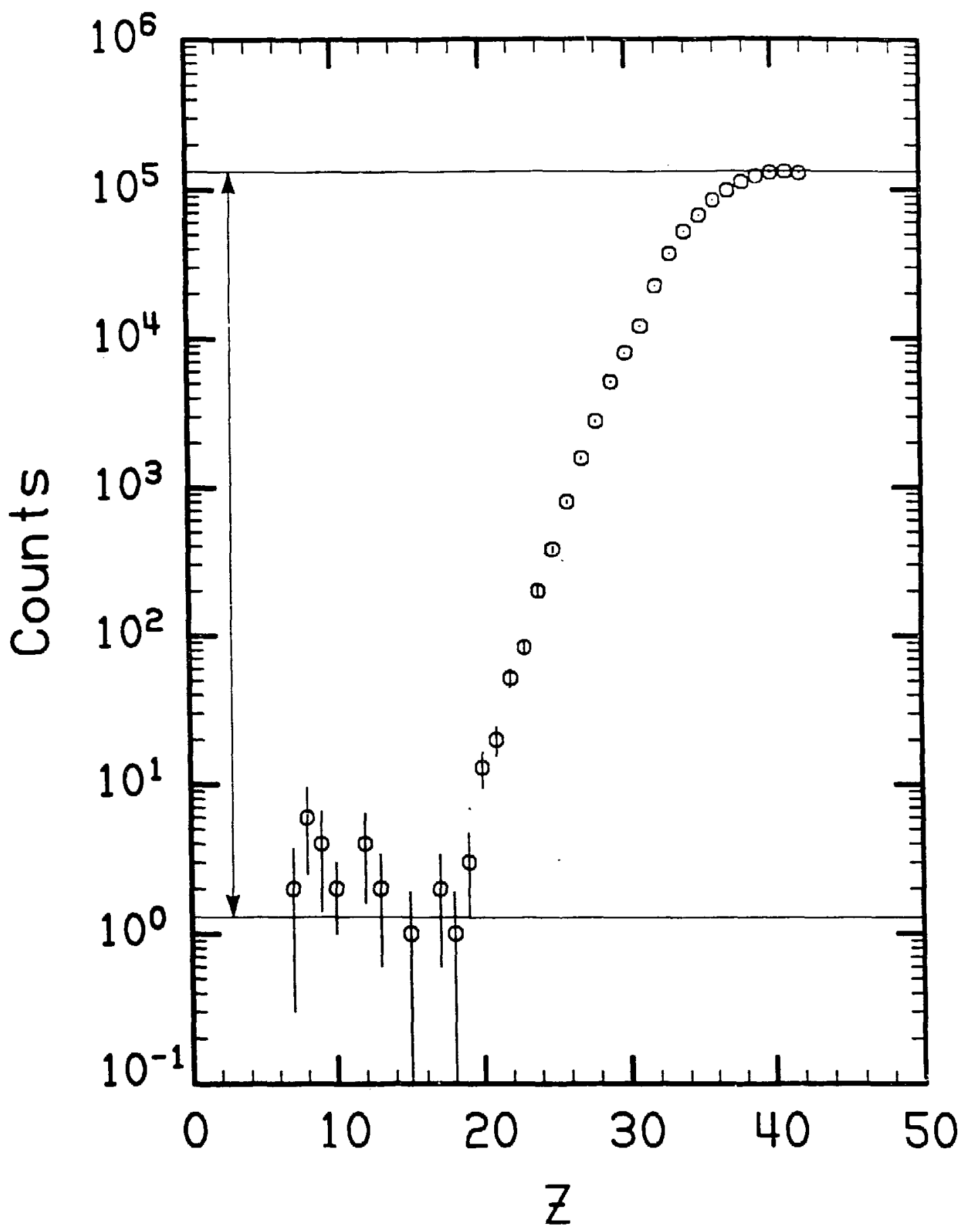

XBL 883.9213

Fig. 3 